\title{
Sorafenib in the Treatment of Virus-Related HCC: Differences Between HCV and HBV
}

\author{
Serena De Matteis' \\ Martina Ghetti ${ }^{2}$ \\ Laura Gramantieri ${ }^{3}$ \\ Giorgia Marisi $\mathbb{D}^{2, *}$ \\ Andrea Casadei-Gardini (iD) ${ }^{4, *}$ \\ 'Department of Specialistic, Diagnostic \\ and Experimental Medicine, University of \\ Bologna, Bologna, Italy; ${ }^{2}$ Biosciences \\ Laboratory, IRCCS Istituto Romagnolo \\ per lo Studio dei Tumori (IRST) "Dino \\ Amadori”, Meldola (FC), Italy; ${ }^{3}$ Center \\ for Applied Biomedical Research, \\ St. Orsola-Malpighi University Hospital, \\ Bologna, Italy; ${ }^{4}$ Department of Medical \\ Oncology, San Raffaele Scientific Institute \\ IRCCS, Milan, Italy
}

*These authors contributed equally to this work
Correspondence: Martina Ghetti Biosciences Laboratory, IRCCS Istituto Romagnolo per lo Studio dei Tumori (IRST) "Dino Amadori", via Piero Maroncelli 40, Meldola (FC), 470I4, Italy Tel/Fax +39-0543-739977

Email martina.ghetti@irst.emr.it

\begin{abstract}
Hepatitis B- and C-virus (HBV and HCV) infections contribute to hepatocellular carcinoma (HCC) development through several different mechanisms. In addition to a diverse molecular background, HCC subtypes also show differences in their metabolic profiles, suggesting that prevention and treatment might require the integration of multiple different approaches. We here analyzed the response of two HCC cell lines representative of different virus-related etiology, namely Hep3B $(\mathrm{HBV}+$ ) and HUH7 (permissive to HCV replication) to sorafenib treatment. Our findings suggest that virus-related specificities influence treatment response in HCC, along with molecular, metabolic and microenvironmental factors. These differences have to be taken into account in the design of future clinical trial aimed to improve HCC patients' outcome.
\end{abstract}

Keywords: hepatocellular carcinoma, hepatitis virus, kinase inhibitor

\section{Introduction}

Hepatitis B- and C-virus (HBV and HCV) infections represent the most common etiology of hepatocellular carcinoma (HCC), leading to progressive chronic inflammation and cirrhosis. The life-cycle of the virus in liver cells differs between HBV and HCV. ${ }^{1} \mathrm{HBV}$ integrates into the liver cell genome, thus contributing to HCC development through several different mechanisms. Conversely, HCV cannot integrate into the host genome. However, it can evade the host immune system and induce genomic instability. ${ }^{2}$ These pathogenic differences between HBV and HCV infection may influence the hepatocyte basal metabolism, culminating in HCC as an end-stage liver disease. It has been previously showed that specific metabolic signatures discriminate HCV- and HBV-related HCC patients. Specifically, serum concentrations of glutamic acid were significantly lower in hepatocellular carcinoma patients $\mathrm{HCV}$ related (HCC-C) than in hepatocellular carcinoma patients HBV related (HCC-B), whereas methionine and $\gamma$-Glu-Gly-Gly levels were significantly higher in HCC-C compared to HCC-B. ${ }^{3}$ Therefore, in addition to molecular features, metabolic profiles differ between HCC subtypes induced by these two viruses, suggesting that prevention and treatment might require different approaches.

Previous studies reported clinical differences in the response to kinase inhibitors. An exploratory subgroup analysis of the SHARP trial showed an increased overall survival (OS) of patients HBV-negative and HCV-positive patients, compared to placebo-group, when treated with sorafenib (median overall survival, 10.7 vs 7.9 months). ${ }^{4}$ Indeed, Kelley et al hypothesized that etiologic factors (such as 
HCV positivity) might be predictive of better response to sorafenib. ${ }^{5}$ These data, confirmed by the pooled analysis of two randomized trials, ${ }^{6}$ suggested that sorafenib may be more effective in HCC-C. In addition, unsupervised transcriptome analysis performed by Boyault et al identified HCC subgroups with different genetic and molecular signatures. $^{7}$ In particular, HCV induced upregulation of
RAF $-1,{ }^{8}$ that is inhibited by sorafenib. This mechanism may contribute to a sorafenib-mediated delay in the progression of HCC-C. Braconi et al highlighted a modulation of microRNAs by HCV proteins that also influenced the sensitivity of HCC cells to sorafenib. ${ }^{9}$ Moreover, Lachaier et al revealed the ability of sorafenib to induce ferroptosis in different cancers including HCC. ${ }^{10}$ Ferroptosis is an

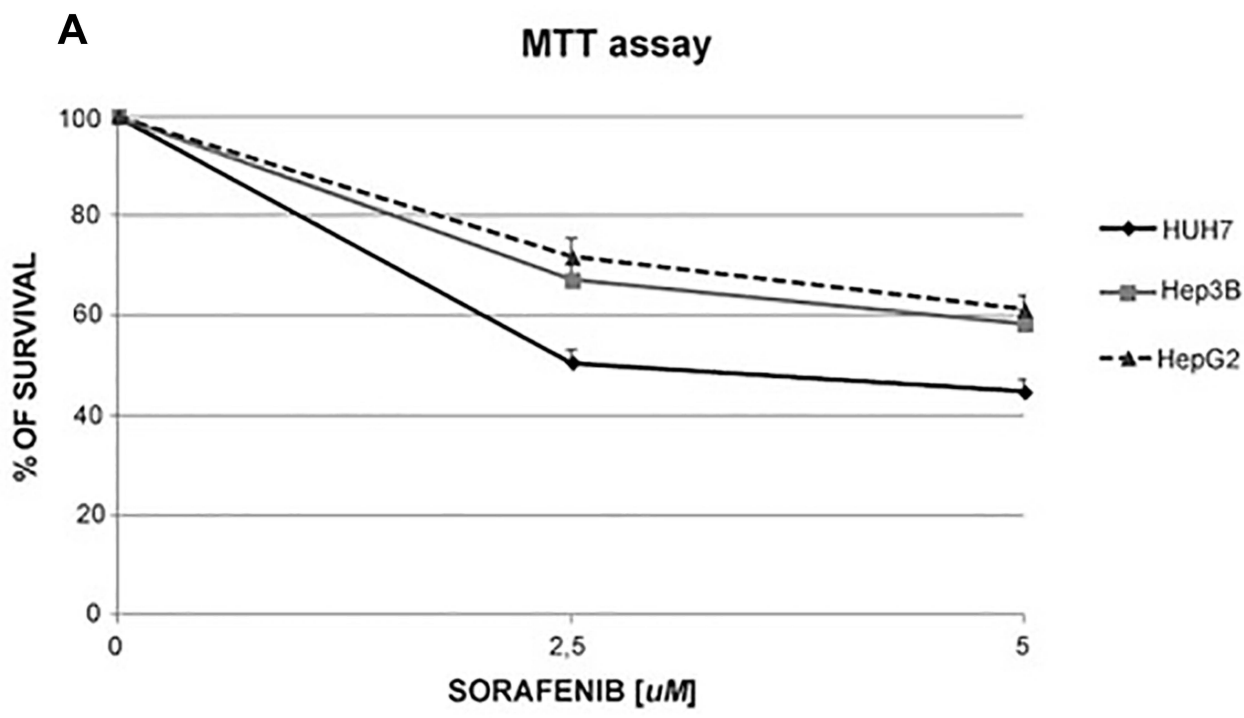

B
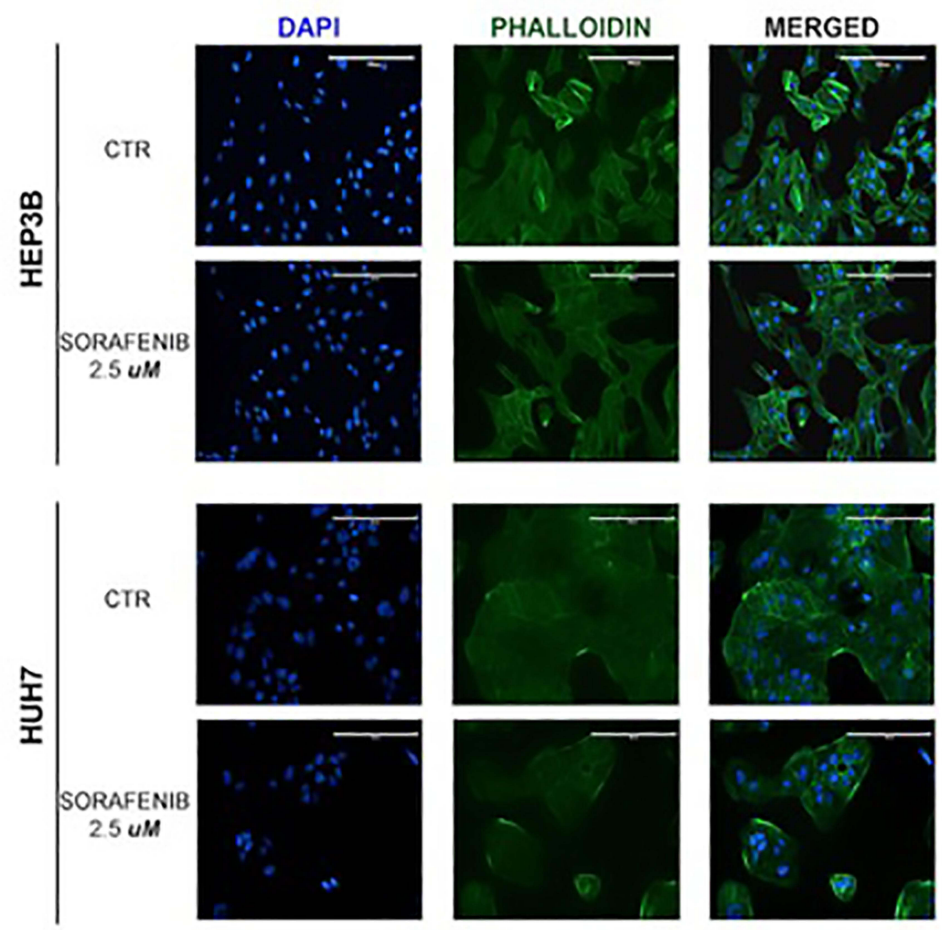

Figure I Sorafenib reduced the cell growth and induced morphological alterations in virus-related HCC cell lines. (A) Survival curves of HCC cell lines treated with different concentrations of sorafenib $(2.5$ and $5 \mu \mathrm{M})$ for $48 \mathrm{~h}$. (B) HCC cell lines were treated with sorafenib at $2.5 \mu \mathrm{M}$ for $48 \mathrm{~h}$, fixed, permeabilized and stained for nuclei with DAPI and for actin with phalloidin. The changes in nuclei and actin filaments were observed by capturing fluorescence images at $20 \times$ magnification. 
iron-dependent, lipid peroxidation-mediated form of cell death, different from apoptosis, necrosis, and autophagy. ${ }^{11}$ Several genes have been reported to regulate the sensitivity of HCC cells to sorafenib by enhancing or inhibiting ferroptosis. ${ }^{12}$ Preclinical data suggested that sorafenib directly inhibits $\mathrm{HCV}$ viral replication. ${ }^{13}$ Conversely to what observed for sorafenib, the analysis of a HCC subgroup in the RESORCE trial ${ }^{14}$ showed a possible benefit of regorafenib in HBV-positive patients compared with HCV-positive ones (HR 0.58, p $=0.0009$ vs HR 0.79, $\mathrm{p}=0.15$; respectively). The same results have been confirmed in the REFLECT trial. ${ }^{15}$

\section{Methods}

Our in vitro study, performed on two HCC cell lines representative of different virus-related etiology, namely Hep3B (HBV+) and HUH7 (permissive to HCV replication), remarked how the $\mathrm{HBV}+$ cell line results less sensitive to treatment with sorafenib than the cell line permissive to $\mathrm{HCV}$ replication. Human HepG2 and Hep3B cell lines were obtained from the American Type Culture Collection (Rockville, MD, USA), whereas the HUH7 were purchased by JCRB Cell Bank (NIBIOHN). Cells were plated onto 96-well microplates and treated with sorafenib at two concentrations $(2,5$ and $5 \mu \mathrm{M})$ for 48 hours. Cell viability was determined by 3-(4,5-dimethylthiazol-2-yl)-2,5-diphenyltetrazolium bromide assay. Cells were then stained with phalloidin and DAPI in order to evaluate the morphologic alterations induced by the treatment.

\section{Results}

Remarkably, a greater reduction of cell proliferation was observed compared to Hep3B cells treated with sorafenib at 2.5 and $5 \mu \mathrm{M}(50 \%$ and $55 \%$ vs $32.79 \%$ and $41.57 \%$, respectively, Figure 1A), as also reported by De Matteis et al. ${ }^{16}$ Notwithstanding, we observed that the HepG2 cell line, that is not virus-related, displays a good response to sorafenib, which is comparable to the one observed for Hep3B (Figure 1A). Moreover, morphological alterations were more evident in the cell line permissive to $\mathrm{HCV}$ replication, when compared to the $\mathrm{HBV}+$ cells. Specifically, HUH7 and Hep3B controls were polygonal and epithelial-like cells. On the contrary, after the treatment with sorafenib at $2.5 \mu \mathrm{M}, \mathrm{HUH} 7$ displayed marked morphological changes, with a rounded shape, whereas Hep3B became spindle-shaped (Figure 1B). It is important to note that the two cell lines have a different origin, with
HUH7 cells being characterized by a stem-like phenotype and a more remarked constitutive activation of the mTOR pathway compared with Hep3B. These features can also affect the response to sorafenib.

\section{Conclusion}

These findings suggest that future randomized trials in HCC, supported by preclinical research, should take into account the genetic, molecular and metabolic differences that characterize the two viruses in order to improve patients' outcome. Consequently, also ferroptosis might have a fundamental impact on the treatment response of HCC.

\section{Abbreviations}

HBV hepatitis B, HCV hepatitis C, HCC hepatocellular carcinoma, OS overall survival.

\section{Disclosure}

Serena De Matteis, Martina Ghetti, Laura Gramantieri, Giorgia Marisi and Andrea Casadei-Gardini declare that they have no conflicts of interest.

\section{References}

1. Baumert TF, Meredith L, Ni Y, et al. Entry of hepatitis B and C viruses - recent progress and future impact. Curr Opin Virol. 2014;4:58-65. doi:10.1016/j.coviro.2013.12.002

2. Ng J, Wu J. Hepatitis B- and Hepatitis C-related hepatocellular carcinomas in the United States: similarities and differences. Hepat Mon. 2012;12(10 HCC):e7635. doi:10.5812/hepatmon.7635

3. Saito T, Sugimoto M, Okumoto K, et al. Serum metabolome profiles characterized by patients with hepatocellular carcinoma associated with hepatitis B and C. World J Gastroenterol. 2016;22:6224. doi:10.3748/wjg.v22.i27.6224

4. Bruix J, Raoul J-L, Sherman M, et al. Efficacy and safety of sorafenib in patients with advanced hepatocellular carcinoma: subanalyses of a Phase III trial. J Hepatol. 2012;57:821-829. doi:10.1016/j. jhep.2012.06.014

5. Kelley RK, Venook AP. Sorafenib in hepatocellular carcinoma: separating the hype from the hope. J Clin Oncol. 2008;26:5845-5848. doi:10.1200/JCO.2008.19.7996

6. Bruix J, Cheng A-L, Meinhardt G, et al. Prognostic factors and predictors of sorafenib benefit in patients with hepatocellular carcinoma: analysis of two phase III studies. J Hepatol. 2017;67:999-1008. doi:10.1016/j.jhep.2017.06.026

7. Boyault S, Rickman DS, de Reyniès A, et al. Transcriptome classification of HCC is related to gene alterations and to new therapeutic targets. Hepatology. 2007;45:42-52. doi:10.1002/hep.21467

8. Giambartolomei S, Covone F, Levrero M, et al. Sustained activation of the Raf/MEK/Erk pathway in response to EGF in stable cell lines expressing the Hepatitis $\mathrm{C}$ virus (HCV) core protein. Oncogene. 2001;20:2606-2610. doi:10.1038/sj.onc.1204372

9. Braconi C, Valeri N, Gasparini P, et al. Hepatitis C virus proteins modulate microRNA expression and chemosensitivity in malignant hepatocytes. Clin Cancer Res. 2010;16:957-966. doi:10.1158/10780432.CCR-09-2123 
10. Lachaier E, Louandre C, Godin C, et al. Sorafenib induces ferroptosis in human cancer cell lines originating from different solid tumors. Anticancer Res. 2014;34(11):6417-6422.

11. Dixon SJ, Lemberg KM, Lamprecht MR, et al. Ferroptosis: an iron-dependent form of nonapoptotic cell death. Cell. 2012;149 (5):1060-1072. doi:10.1016/j.cell.2012.03.042

12. Feng H, Schorpp K, Jin J, et al. Transferrin receptor is a specific ferroptosis marker. Cell Rep. 2020;30(10):3411-3423.e7. doi:10.1016/j.celrep.2020.02.049

13. Himmelsbach K, Sauter D, Baumert TF, et al. New aspects of an anti tumour drug: sorafenib efficiently inhibits $\mathrm{HCV}$ replication. Gut. 2009;58:1644-1653. doi:10.1136/gut.2009.182212
14. Bruix J, Qin S, Merle P, et al. Regorafenib for patients with hepatocellular carcinoma who progressed on sorafenib treatment (RESORCE): a randomised, double-blind, placebo-controlled, Phase 3 trial. Lancet. 2017;389:56-66. doi:10.1016/S0140-6736(16)32453-9

15. Kudo M, Finn RS, Qin S, et al. Lenvatinib versus sorafenib in first-line treatment of patients with unresectable hepatocellular carcinoma: a randomised phase 3 non-inferiority trial. Lancet. 2018;391:1163-1173. doi:10.1016/S0140-6736(18)30207-1

16. De Matteis S, Scarpi E, Granato A, et al. Role of SIRT-3, p-mTOR and HIF- $1 \alpha$ in hepatocellular carcinoma patients affected by metabolic dysfunctions and in chronic treatment with metformin. Int J Mol Sci. 2019;20:1503. doi:10.3390/ijms20061503

\section{Publish your work in this journal}

OncoTargets and Therapy is an international, peer-reviewed, open access journal focusing on the pathological basis of all cancers, potential targets for therapy and treatment protocols employed to improve the management of cancer patients. The journal also focuses on the impact of management programs and new therapeutic

Submit your manuscript here: https://www.dovepress.com/oncotargets-and-therapy-journal agents and protocols on patient perspectives such as quality of life, adherence and satisfaction. The manuscript management system is completely online and includes a very quick and fair peer-review system, which is all easy to use. Visit http://www.dovepress.com/ testimonials.php to read real quotes from published authors. 